\title{
Nervous system and Fabry disease, from symptoms to diagnosis: damage evaluation and follow-up in adult patients, enzyme replacement, and support therapy
}

\author{
Alessandro Salviati • Alessandro P. Burlina • \\ Walter Borsini
}

Received: 30 March 2009/Accepted: 16 December 2009/Published online: 19 March 2010

(C) The Author(s) 2010. This article is published with open access at Springerlink.com

\begin{abstract}
The X-linked genetic Fabry disease causes multiorgan lesions due to intracellular storage of the substrate globotriaosylceramide. Neurological involvement ranges from painful, small fiber neuropathy to cerebrovascular disorders to multifocal aggressive forms. Disease identification through proper differential diagnosis and timely assessment of organ damage should guide a careful treatment planning. Mainstay treatment, include enzyme replacement and support therapy. Neurologists have a pivotal role in early instrumental and clinical detection of organ damage. A panel of experts has developed a set of consensus recommendations to guide the approach of neurologists to Fabry disease.
\end{abstract}

Keywords Fabry - Enzyme replacement therapy · Small fiber neuropathy - Globotriaosylceramide

A. Salviati, A. P. Burlina, and W. Borsini contributed equally to this work.

This paper is the result of a consensus development process initiated in 2007. Four meetings were held in Italy. The consensus group consisted of different Italian specialists in Fabry disease. All specialists reviewed the literature in the specific field and prepared a document of standard care which has been discussed in the plenary sections. The neurological group comprised the authors.

\section{A. Salviati}

Section of Clinical Neurology,

Department of Neurological Sciences and Vision,

University Hospital, Verona, Italy

\section{A. P. Burlina ( $\bowtie)$}

Neurological Unit, San Bassiano Hospital,

Via dei Lotti 40, 36061 Bassano del Grappa, Italy

e-mail: alessandro.burlina@ aslbassano.it

W. Borsini

Department of Neuroscience, Careggi Hospital,

University of Florence, Florence, Italy

\section{Definition}

Fabry disease is an X-linked lysosomal storage disorder caused by the deficiency of the enzyme $\alpha$-galactosidase $A$ $(\alpha$-Gal A). Intracellular accumulation of the substrate globotriaosylceramide (Gb3) leads to multiorgan lesions $[1,2]$. Fabry disease affects men, but it is often found in heterozygous women as well.

\section{Clinical pictures and course}

The classic form of Fabry disease is generally observed in male patients in the pediatric age group. Symptoms are sometimes not recognized until the youth or adult age, when organ lesions are already established. The earliest manifestations of disease, painful neuropathy and angiokeratoma, are observed in the majority of patients [3-6]: 72 , and $88 \%$, respectively, in our experience [7]. The onset of painful neuropathy is observed on average around the eleventh year of life, but it has also been reported in earlier years. Gastrointestinal disturbances in young patients are not rare. Cornea verticillata without alterations of vision is a very frequent finding. Later, in adult men between 30 and 40 years, complications arise involving vital organs, i.e., the heart, central nervous system, and kidney (altered renal function ranges from proteinuria to progressive renal failure leading to kidney transplant). Neurosensorial hearing loss and paroxysmal vertigo can also be observed; the latter may be an indication of labyrinth damage. Life expectancy was about 41-50 years before enzyme replacement therapy (ERT) and kidney transplant were available [3, 4, 6]. In women, the clinical course is less aggressive, with onset later in adult life. In women, and more rarely in men with atypical clinical pictures, manifestations involving only 
one organ are paramount (e.g., central nervous system or the heart, while angiokeratoma has only limited extension or it is not referred to the physician by the patient, and proteinuria is mild or absent), and painful neuropathy seems to have a lower incidence than in men. The average onset of painful neuropathy in women is around age 17 years, but it may also be observed in earlier years of life. The Fabry registry reports a median age of onset at 10 years. Life expectancy in these patients is 55-70 years, lower than the average life expectancy in women in the general population $[3,4,6]$.

\section{From clinical hypothesis to diagnosis of Fabry disease: opportunities for the neurologist}

Early diagnosis is important to establish specific (i.e., ERT) and symptomatic and preventive therapy. Clinical suspicion of Fabry disease, if clinical pictures as those described should occur, can be validated by clinical history, physical examination with special attention to specific signs, and by symptoms not spontaneously reported by the patient, such as angiokeratoma and heat intolerance. Proteinuria, ECG alterations (shortage of the PR interval), cornea verticillata, and echocardiographic alterations suggestive of hypertrophic myocardiopathy are important clues for the disease. However, such signs and symptoms may be absent or not clearly defined in typical manifestations of the disease and they are easily missed unless carefully sought for. When suspecting Fabry disease, diagnosis is achieved as follows: in men through a leukocyte $\alpha$-galactosidase A assay (in typical forms, activity is low or absent) later confirmed by molecular genetic analysis; in women, DNA analysis is strongly required. In women, leukocyte enzyme activity shows strong inter-individual variability, ranging from normal to significantly reduced values, probably because of random inactivation of one of the $\mathrm{X}$ chromosomes in the early stages of development. Central and peripheral nervous system symptoms may also have diagnostic value, and it is worth alerting the general practitioner and the pediatrician of the changing clinical features of nervous system involvement in Fabry disease [7-11]:

1. painful neuropathy (small fiber neuropathy);

2. cerebrovascular disorders (small and large vessel disease and/or cardiovascular involvement with cerebral embolism);

3. aggressive clinical forms with central multifocal, relapsing, and progressively invalidating symptoms, with possibly superimposed inflammatory conditions (aseptic meningitis, multiple sclerosis-like syndromes).
Involvement of peripheral nerves, with neuropathic pain and acroparesthesia, which is triggered or worsened by temperature increase, is the most frequent and early manifestation of Fabry disease, and often the first one. These symptoms are due to a dysfunctional condition of small caliber nervous fibers [12-14].

Symptoms described above are observed in about $80 \%$ of male patients, more rarely in women, and appear early. In adults, symptoms may also be induced by stress and alcohol intake. Upper and lower limbs are involved, particularly the palm of the hands and the foot sole, while the skin color is not affected and such symptoms are always associated with paresthesia, mainly thermal sensitivity, while deep tendon reflexes are preserved. Severity is variable among patients; and variable in the same patient over time. Nerve conduction studies (NCS) can be normal even in the late stages of the disease, because small fiber involvement is the predominant feature of the disease. The incidence of the carpal tunnel syndrome looks higher than in the general population [13]. Owing to NCS results, small fiber neuropathy may be difficult to substantiate. Skin biopsy, revealing loss of axons and osmophilic inclusions, may be useful in selected cases [15, 16]. Involvement of the autonomous nervous system is frequent, and should carefully be looked (Table 1). Gastrointestinal manifestations of autonomous nervous system involvement may range from abdominal pain to diarrhea and rarely stypsis; in women abdominal pain may be considered, by mistake, of gynecological origin. Altered sweating function is a frequent and classic feature. High temperature increases poor tolerance: fever, and high environmental temperature, as well as physical exercise can trigger acute pain at the extremities with weakness, which are often intense, and generalized malaise (thermoregulation through sweating is not effective in Fabry disease). Impaired autonomic function with altered thermoregulatory sweating causes such symptoms; such symptoms can also be seen with low environmental temperature. Either hypotension (more often) or hypertension may also be seen.

Table 1 Other conditions leading to painful neuropathy besides Fabry disease

Differential diagnosis of painful neuropathy in Fabry disease

1. Juvenile rheumatoid arthritis

2. Pain from other rheumatic disorders

3. Pain commonly attributed to growth

4. Painful neuropathy in other inherited metabolic conditions (e.g., amyloidosis and porphyria)

5. Painful neuropathy in other metabolic conditions (e.g., diabetes mellitus type I, alcohol abuse, deficiency of vitamins)

6. Neuropathies in systemic vasculitides

7. Psychogenic pain 
Cochleovestibular disorders are also common, especially hypoacusia [17]. Cerebrovascular ischemic events (brain infarctions or TIA) are present in over $25 \%$ of patients, and their frequency increases with age. Involved areas are mostly, although not exclusively, those supplied by the posterior arterial circulation. An etiology of each single event is hard to identify, and it is likely to be mixed even in the same patient at different times: arteriolar obstruction, arteriosclerosis, arterio-arterial embolism and cardiogenic embolism are all potential mechanisms. Magnetic resonance imaging (MRI) is useful to assess nervous system lesions. Typically, MRI may show lesions attributable to small infarctions and diffuse alteration of the white matter, especially in deeper sections, with images suggestive of arteriolar involvement of the perforating arteries (lacunar infarctions and leukoaraiosis). These lesions are often silent, and they are discovered at early stages in adult patients undergoing screening and follow-up. In some instances, abnormalities of brain MRI related to small vessel pathology (white matter lesions) are the only signs leading to diagnosis of Fabry disease [18, 19]. Characteristic, but non specific, neuroradiological features of Fabry disease have been found almost exclusively in men. Indeed, bilateral T1 hyperintensity can be seen in the posterior region of thalamus ("pulvinar sign") in male patients [20]. Except for the "pulvinar sign" differential neuroradiological diagnosis of Fabry's CNS lesions with other vascular pathologies or inflammatory disorders can be rather difficult. Vertebro-basilar arterial dolichoectasy is very common in neuroimaging studies (38\% in men, $57 \%$ in women according to Rolfs et al., up to $87 \%$ in the report of Fellgiebel et al.) [21, 22], even relatively early in the course of the disease with the endothelial thickness still normal. Transcranial Doppler data provides not conclusive results in Fabry patients, and it still has no role in the clinical management of individual cases [23, 24]. Fabry disease should always be considered in the differential diagnosis of stroke in young age, even without clinical evidence of cardiac or renal involvement. A recent prospective study suggests that $4 \%$ of cryptogenetic strokes in the youth are attributable to Fabry disease, and 1-2\% of patients with stroke between 18 and 55 years have Fabry disease [21]. Table 2 shows a list of clinical conditions for the differential diagnosis with Fabry disease. Etiology of stroke may be cardiac (cardiomyopathy, valvulopathy, arrythmias), hypotensive or it may follow deposition of GL3 in vessel walls. Embolic strokes have been described in elderly women with Fabry disease with cardiomyopathy and atrial fibrillation [25]. Glial scars following cortical necrosis in patients with vascular encephalopathy may be epileptogenic and cause seizures, albeit rarely. Cerebrospinal examination (CSF),
Table 2 Other conditions, leading to stroke and vascular encephalopathy, which should be differentiated from Fabry disease

Stroke and vascular encephalopathy in Fabry disease: differential diagnosis

1. Juvenile cryptogenetic ischemic stroke

2. Juvenile ischemic stroke associated with other acquired risk factors

3. Juvenile stroke in monogenic diseases (e.g., CADASIL, homocystinuria)

4. Mitochondrial diseases (e.g., MELAS)

5. Central nervous system and systemic vasculitis

6. Multifocal/lacunar leukoencephalopathy of unspecified etiology

7. Amyloidosis

8. Demyelinating diseases (CSF examination)

sometimes suggesting an altered blood-brain barrier (BBB), is helpful in discriminating among different putative causes.

Albeit rarely, acute and recurrent cerebrovascular events may associate with aseptic meningitis, and CSF concentration of proteins are increased due to an altered brainblood barrier [10]. In one case, a beneficial effect of highdose steroids has been reported [10].

\section{Early evaluation of Fabry disease-related organ damage and clinical course}

Instrumental diagnosis allows for early detection of organ damage before symptoms are established. Early detection of organ damage is important because any treatment will have more chances of success if started before irreversible structural organ damage develops $[7,26]$. Structural, irreversible organ damage can develop early in the asymptomatic stage, but it is more extensive and diffuse in the symptomatic stages of the disease. Direct correlation of organ damage with Fabry disease should be documented by biopsy whenever applicable. Of course, even when there is no such direct evidence, clinical and instrumental investigations will lead the expert physician to establish the correlation between organ damage and Fabry disease. Pathogenesis of organ damage is multifactorial in Fabry disease as in many other monogenic diseases, and that would explain the heterogeneity of phenotypic manifestations within the same family and in individuals of the same sex and age [27]. Multifactorial pathogenesis is of special value to explain how vasculopathy, with involvement of small and large vessels, originates in Fabry disease. Early documentation of organ damage, in addition to diagnosis, is important in clinical practice to address treatment options. Neurological signs and symptoms, even though they are limited to peripheral neuropathy, frequently 
trigger the search for medical assistance, and they may be crucial in guiding treatment. Organ involvement should be monitored in male and female Fabry patients, ideally in specialized healthcare facilities. The following evaluations are useful in monitoring peripheral and central nervous system involvement in male and female patients, even in asymptomatic patients and in patients who are not undergoing treatment:

1. neurological assessment;

2. disability and quality of life evaluation, e.g. with Rankin scale [28], Euro-Qol [29], and Mainz Severity Score Index or MSSI [30], together with the traditional SF-36 quality of scale, which is used in the Fabry Registry;

3. non contrast-enhanced MRI of the brain with MRI angiography of intracranial vessels;

4. diagnostic ultrasonography of neck vessels;

5. examination and evaluation for cardiovascular riski.e., combination of common acquired or genetic risk factors.

Clinical course will determine frequency of tests and clinical evaluation. Annual evaluations are also warranted for asymptomatic individuals, especially in women, for early detection of central nervous system involvement. In patients undergoing ERT, recommended examinations should be performed according to clinical opportunity, or at least once a year in the first 2 years of treatment.

Other examinations, which are useful for the neurologist, are

1. NCS (usefulness is limited, but it can reveal the coexistence of other neuropathic conditions such as kidney failure or dialysis-associated polyneuropathy);

2. skin biopsy for studying intraepidermic innervation (skin biopsy may be also warranted to look for indications of small fiber neuropathy).

\section{Enzyme replacement therapy}

Specific pharmacologic therapy for Fabry disease with ERT is endorsed by health regulatory agencies. Two authorized drugs are available in Europe for ERT in Fabry disease. Characteristics and efficacy data of the two drugs are summarized in two recent articles [31, 32]; the management of multiorgan involvement is described in Eng et al. [33]. Both are administered intravenously, with one infusion every 14 days. Agalsidase beta $\left(\right.$ Fabrazyme $^{\circledR}$ ) is approved for maintenance regimens of $1 \mathrm{mg} / \mathrm{kg}$ every 14 days. A dose of $0.3 \mathrm{mg} / \mathrm{kg}$ (after a 24-month $1 \mathrm{mg} / \mathrm{kg}$ treatment and depending on assessment of clinical course) may maintain GL-3 clearance in some patients with Fabry disease, but other patients seem to require a dose higher than $0.3 \mathrm{mg} / \mathrm{kg}$ to prevent recurrence of GL-3 accumulation in cells. The long-term clinical effects of transitioning to the lower dose were not evaluated. Nevertheless, the results clearly show variability in responsiveness to the lower dose of agalsidase beta and suggest that dosing strategies that deviate from the recommended therapeutic regimen of $1.0 \mathrm{mg} / \mathrm{kg}$ every 2 weeks should be systematically monitored [34]. Agalsidase alpha (Replagal ${ }^{\circledR}$ ) is approved for maintenance regimens of $0.2 \mathrm{mg} / \mathrm{kg}$ every 14 days.

Evidence of efficacy is available for each product [3439] and dosage. However, disease progression is also reported with both drugs, at least in the first years of ERT in patients in late clinical stages and with organ damage [7, 26]. Both synthetic enzymes do not cross the BBB. Treatments can, therefore, only act on the endothelial cells of the cerebral arterial circulation, at least when the BBB is intact (in aggressive or MS-like and aseptic meningitis-like forms of Fabry disease with lacunar infarcts, BBB may be seriously altered). At present, long longitudinal studies with adequate number of enrolled patients are not yet available to support evidence of efficacy of ERT in preventing new cerebrovascular events.

Young or adult Fabry disease patients with a genetic mutation leading to a lack or marked reduction in leukocyte alpha-galactosidase A are eligible to undergo ERT even with simple evidence of angiokeratoma and/or painful neuropathy and before the onset of signs of vital organ damage (male patients with the classic form of the disease). Prognosis for this group of patients is actually rather predictable, with high likelihood of developing terminal renal failure around age of 40 years. Fabry male patients with atypical clinical features-residual leukocyte alpha-galactosidase A activity, prevalent cardiovascular involvement, lengthy clinical course and lack of evidence of angiokeratoma, painful polyneuropathy-could be treated when relevant signs and symptoms are present. This more cautious conduct in initiating treatment is justified by lack of definite knowledge about the long-term prognosis of these patients. ERT is indicated in women as well, but guidelines published in other countries (Great Britain, Australia, Canada), and also guidelines by an expert panel [33] restrict its use to women with clinical signs or symptoms, always because of difficulties in predicting the outcome in the individual patient. In the literature, there are several evidences of symptoms improvement, as well as reports of improvement of some indicators of painful neuropathy and of hypo/ anhydrosis [40-42]. These latter signs are also supported by clinical evidence, even if there is no evidence of intraepidermic nerves regeneration [43]. As a matter of fact, usually neuropathic pain and hypo/anhydrosis do respond to ERT, either in men either in women. In male patients, such symptoms are frequent, almost always in combination with 
overt multiorgan clinical manifestations, while in women neuropathic pain can either be an isolated symptom or it can be one of the major symptoms. The follow-up is always important for assessing the real impact of ERT on neuropathic pain. Evidence of a favorable impact of ERT on central nervous system signs and symptoms has not yet been established. All favorable data, e.g., functional modifications of cerebral arterial circulation, assessed mainly with transcranial Doppler, are still under discussion and interpretation [23, 24]. Furthermore, documented structural alterations of cerebral parenchyma and/or cerebral vessels must be considered as non-early complications. Decision to start ERT, especially in men, is, therefore, rarely taken based only on the involvement of the nervous system, except for the following circumstances:

1. rare, very aggressive forms in young men with predominant CNS symptoms and not much evidence of classic signs (neuropathy, angiokeratoma);

2. women and men with atypical presentation, with prevalent clinical manifestation and/or neuroradiological specific involvement of central nervous system (TIA or stroke; subclinical MRI abnormalities, i.e. white matter changes), even when onset is late (e.g., 40-50 years).

ERT is authorized in Europe for all confirmed Fabry patients, regardless of organ involvement and clinical severity. For patients with prevalent central nervous system involvement, ERT should be prescribed by specialized physicians taking into consideration the following conditions:

1. without a confirmatory kidney biopsy, the medical team should ascertain that Fabry disease has a pathogenetic role. Finding of central nervous system MRI preclinical abnormalities, when correctly assessed by an expert team, may indicate a precocious stage of the disease, which could benefit of an early treatment, even though we do not have yet clinical studies which demonstrate such a result;

2. the patient must be informed of our limited current knowledge on this specific topic;

3. careful monitoring of the clinical course of disease and outcome of treatment are mandatory;

4. it must be kept in mind that cerebrovascular manifestations of Fabry disease often have a multifactorial pathogenesis-i.e., genetic and acquired risk factors for cerebrovascular disease and multiorgan involvement (cardiovascular and renal damage), including alterations of blood pressure regulation with arterial hypo-hypertension, both directly or indirectly linked to Fabry disease. Therefore, support therapy, i.e. antiplatelet or anticoagulant treatment and care of potentially associated cerebrovascular risk factors, should always be started together with the ERT.

\section{Home-based ERT}

Enzyme replacement therapy is a lifelong therapy. As home-based therapy, it could be beneficial for quality of life and optimization of healthcare resources. Some studies reported that home-based therapy is feasible and advantageous for both patients and the healthcare systems, provided that the patient is stable; his dwelling is suitable, community-based services are adequate, and intervention protocols for treatment of potential reaction associated with the infusion are in place [44-46].

\section{Symptomatic and preventive therapy}

Kidney transplants have changed life perspectives of Fabry patients. Other important preventive and support treatments, include antiplatelet drugs, anticoagulants, antiarrhythmics, ICDs, pacemaker, ACE inhibitors for proteinuria, and dialysis. Moreover, prevention measures addressing multifactorial vascular risk also have a key role in Fabry patients-e.g., treatments for hypertension. Gabapentin and carbamazepine may be effective symptomatic treatments for neuropathic pain [47, 48]; such treatments are often carried out in the setting of clinical teams including many specialists. Satisfactory results, albeit limited in time, have been recently reported in aggressive CNS involvement (MS-like forms or aseptic meningitis with lacunar infarctions) with bolus high-dose cortisone [49]; the authors also observed temporary beneficial effects of corticosteroid treatment in these cases.

Conflict of interest statement The authors declare no conflict of interest whatsoever.

Open Access This article is distributed under the terms of the Creative Commons Attribution Noncommercial License which permits any noncommercial use, distribution, and reproduction in any medium, provided the original author(s) and source are credited.

\section{Appendix: neurologic group consensus: early diagnosis of Fabry disease}

\footnotetext{
Recommendation 1

Suspected Fabry disease should be confirmed as follows

Leukocyte alpha-galactosidase A activity assay in men (in the classic form of disease, enzyme activity is always very low or absent)

In women, and more rarely in men with residual enzyme activity, molecular analysis of GLA gene must be performed
} 


\section{Recommendation 2}

Recognize the variable nervous system involvement in Fabry disease

Painful neuropathy (small fiber neuropathy)

Cerebrovascular disorders (small and large vessels and/or cardiovascular disorders with cerebral embolism)

Aggressive forms with multifocal, relapsing central symptoms, and progressive disability (multiple sclerosis-like and/or aseptic meningitis)

\section{Recommendation 3}

Recognize painful neuropathy of Fabry disease for early diagnosis

Painful neuropathy

Early onset (usually childhood), especially in men

Association between neuropathic pain and retained tendon reflexes and normal NCS

Association with intolerance to extreme temperatures and hypo/ anhydrosis

Small fiber neuropathy (skin biopsy)

\section{Recommendation 4}

Fabry disease is often unrecognized as a cause of TIA/stroke

\section{$>$ TIA/stroke}

In young men, and in women or in men with atypical forms, aged $40-50$ years

Cerebral embolism in patients with atrial fibrillation, even at age $>50$ years

Association with intolerance to extreme temperatures and hypo/ anhydrosis

Association with one or more of the following clinical features as angiokeratoma, cornea verticillata, proteinuria, and painful neuropathy in women and men

Should TIA/stroke or brain MRI signs confirm small vessel disease in a patient aged 55 years or younger, consider Fabry disease. Brain MRI (Flair sequence) detects small cerebral vessels alteration with high sensitivity, but it lacks specificity

\section{Documenting multiorgan damage}

Recommendation 5

Validate diagnosis of organ damage

Early documentation of organ damage in patients with confirmed diagnosis is currently considered an important feature to guide treatment in clinical practice

Attribute a score to the extent of damage in the peripheral nervous system

Neurologists will document peripheral nervous system involvement

\section{Appendix continued}

Scoring systems are used to assess neuropathic pain and monitor changes (visual analog scale or VAS, Brief Pain Inventory or BPI [50], McGill Pain Questionnaire) [51]

Skin biopsy with study of intraepidermic innervation should currently be reserved to specific cases

NCS has a role in differential diagnosis with large fiber neuropathy, which may also be seen in Fabry disease (e.g., with progression of kidney failure)

\section{Recommendation 6}

Useful tests to monitor cerebrovascular disorders in patients with Fabry disease

Recommended investigations for early evaluation and monitoring of CNS damage

Anamnesis, genetic family tree, and neurological examination

Assessment of general health status (Rankin, EuroQoL, SF-36, WHOQoL-100)

Brain MRI (including T1 sequence for identification of the pulvinar sign, and intracranial MRI angiography)

Diagnostic ultrasonography of epiaortic vessels

Cardiologic evaluation, EKG and echocardiography

Assessment of modifiable cardiovascular risk factors (e.g., homocysteinemia, folate serum levels, etc.)

Biopsy for vasculitis

Clinical evaluation suggests timing of examinations (at least yearly)

Monitoring of ERT patients on ERT should be performed with the same tests, which will be repeated at least once a year for the first 2 years of treatment or according to clinical considerations

\section{Therapy}

\section{Recommendation 7}

Health Regulatory Agencies recommend ERT in confirmed Fabry disease

Any decision on treatment should be made by considering the full clinical presentation

Due to reduction in life expectancy, affected males should start ERT even during adolescence if angiokeratoma and/or painful neuropathy are reported

Male patients with atypical clinical forms (with residual leukocyte alpha-galactosidase A, absence of angiokeratoma and painful polyneuropathy, slower clinical progression) should be treated at onset of organ involvement, even without related symptoms

Not all affected women should be treated, but only those with involvement of vital organs or significant neuropathic pain

ERT is prescribed in clinical settings specialized in the diagnosis and care of Fabry disease 
Recommendation 8

Enzyme replacement therapy is effective in the treatment of painful neuropathy

Assess what symptoms are due to Fabry disease

Assess relevance of symptoms for the patient's quality of lifemainly in women with only one symptom or sign

Make sure the patient is adequately informed on treatment and compliance is maintained

Carry out correctly the patient's follow-up

Identify a symptomatic drug for the treatment of the neuropathic pain (e.g., gabapentin)

\section{Recommendation 9}

Efficacy of enzyme replacement therapy on central nervous system involvement is currently not established

Further investigations are required

If ERT is started in symptomatic patients, progression of disease may still occur

Neurologists should focus on preclinical detection of CNS involvement

If ERT is prescribed for central nervous system involvement, as may be the case for women, a decision, carried out by a team of experts, for any single patient is recommended with the following goals

Assessing that symptoms to Fabry disease are correctly identified

Designing a multitarget treatment (for prevention of cardio- and cerebrovascular risks)

Evaluating awareness and compliance of patient with the treatment program

Carrying out correctly the patient's follow-up

\section{References}

1. Brady RO, Gal AE, Bradley RM, Martensonn E, Warshaw AL, Laster L (1967) Enzymatic defect in Fabry's disease: ceramidetrihexosidase deficiency. New Engl J Med 276:1163-1167

2. Desnick RJ, Ioannou YA, Eng ME (2001) $\alpha$-Galactosidase A deficiency: Fabry disease. In: Scriver CR, Beaudet AL, Sly WS, Valle D (eds) The metabolic and molecular basis of inherited disease, vol 3, 8th edn. McGraw-Hill, New York, pp 3733-3774

3. MacDermot KD, Holmes A, Miners AH (2001) Anderson-Fabry disease: clinical manifestations and impact of disease in a cohort of 98 hemizygous males. J Med Genet 38:750-760

4. Mehta A, Widmer U (2006) Natural history of Fabry disease. In: Mehta A, Beck M, Sunder-Plassmann G (eds) Fabry disease: perspectives from 5 years of FOS. Oxford Pharmagenesis, Oxford, pp 183-188

5. Mehta A, Ricci R, Widmer U, Dehout F, Garcia de Lorenzo A, Kampmann C, Linhart A, Sunder-Plassmann G, Ries M, Beck M (2004) Fabry disease defined: baseline clinical manifestations of 336 patients in the Fabry outcome survey. Eur J Clin Invest 34:236-242

6. Eng CM, Fletcher J, Wilcox WR, Waldek S, Scott CR, Sillence DO, Breunig F, Charrow J, Germain DP, Nicholls K, Banikazemi M (2007) Fabry disease: baseline medical characteristics of a cohort of 1765 males and females in the Fabry Registry. J Inherit Metab Dis 30:184-192

7. Buechner S, Moretti M, Burlina AP, Cei G, Manara R, Ricci R, Mignani R, Parini R, Di Vito R, Giordano GP, Simonelli P, Siciliano G, Borsini W (2008) Central nervous system involvement in Anderson-Fabry disease: a clinical and MRI retrospective study. J Neurol Neurosurg Psychiatry 79:1249-1254

8. Fellgiebel A, Müller MJ, Ginsberg L (2006) CNS manifestations of Fabry's disease. Lancet Neurol 5:791-795

9. Møller AT, Jensen TS (2007) Neurological manifestations in Fabry's disease. Nat Clin Pract Neurol 3:95-106

10. Lidove O, Chauveheid MP, Caillaud C, Froissart R, Benoist L, Alamowitch S, Doan S, Szalat R, Baumann N, Alexandra JF, Lavallée P, Klein I, Vuillemet F, Sedel F, Sacré K, Samson Y, Roullet E, Papo T (2009) Aseptic meningitis and ischemic stroke in Fabry disease. Int J Clin Pract 63:1663-1667

11. Invernizzi P, Bonometti MA, Turri E, Benedetti MD, Salviati A (2008) A case of Fabry disease with central nervous system (CNS) demyelinating lesions: a double trouble? Mult Scler 14:1003-1006

12. Clavelou P, Besson G (2007) Neurological aspects of Fabry disease. Presse Med 36 Spec No 1:1S65-S68

13. Schiffmann R (2009) Fabry disease. Pharmacol Ther 122:65-77

14. Low M, Nicholls K, Tubridy N, Hand P, Velakoulis D, Kiers L, Mitchell P, Becker G (2007) Neurology of Fabry disease. Intern Med J 37:436-447

15. Laaksonen SM, Röyttä M, Jääskeläinen SK, Kantola I, Penttinen M, Falck B (2008) Neuropathic symptoms and findings in women with Fabry disease. Clin Neurophysiol 119:1365-1372

16. Liguori R, Di Stasi V, Bugiardini E, Mignani R, Burlina A, Borsini W, Baruzzi A, Montagna P, Donadio V (2009) Small fiber neuropathy in female patients with Fabry disease. Muscle Nerve 41:409-412

17. Conti G, Sergi B (2003) Auditory and vestibular findings in Fabry disease: a study of hemizygous males and heterozygous female. Acta Paediatr 92(Suppl 443):33-37

18. Fellgiebel A, Müller MJ, Mazanek M, Baron K, Beck M, Stoeter $\mathrm{P}$ (2005) White matter lesion severity in male and female patients with Fabry disease. Neurology 65:600-602

19. Ginsberg L, Manara R, Valentine AR, Kendall B, Burlina AP (2006) Magnetic resonance imaging changes in Fabry disease. Acta Paediatr 95(Suppl 451):57-62

20. Burlina AP, Manara R, Caillaud C, Laissy JP, Severino M, Klein I, Burlina A, Lidove O (2008) The pulvinar sign: frequency and clinical correlations in Fabry disease. J Neurol 255:738-744

21. Rolfs A, Böttcher T, Zschiesche M, Morris P, Winchester B, Bauer P, Walter U, Mix E, Löhr M, Harzer K, Strauss U, Pahnke J, Grossmann A, Benecke R (2005) Prevalence of Fabry disease in patients with cryptogenic stroke: a prospective study. Lancet 366:1794-1796

22. Fellgiebel A, Keller I, Marin D, Müller MJ, Schermuly I, Yakushev I, Bellhäuser H, Kinateder M, Beck M, Stoeter P (2009) Diagnostic utility of different MRI and MR angiography measures in Fabry disease. Neurology 72:63-68

23. Moore DF, Altarescu G, Ling GS, Jeffries N, Frei KP, Weibel T, Charria-Ortiz G, Ferri R, Arai AE, Brady RO, Schiffmann R (2002) Elevated cerebral blood flow velocities in Fabry disease with reversal after enzyme replacement. Stroke 33:525-531

24. Hilz MJ, Kolodny EH, Brys M, Stemper B, Haendl T, Marthol H (2004) Reduced cerebral blood flow velocity and impaired cerebral autoregulation in patients with Fabry disease. J Neurol 251:564-570

25. Buechner S, Luzzi C, Mannucci M, Massi D, Borsini W (2006) Diagnosis of Anderson-Fabry's disease in over seventy yearold women: description of two cases. Aging Clin Exp Res 18:340-343 
26. Wilcox WR, Banikazemi M, Guffon N, Waldek S, Lee P, Linthorst GE, Desnick RJ, Germain DP, International Fabry Disease Study Group (2004) Long-term safety and efficacy of enzyme replacement therapy for Fabry disease. Am J Hum Genet 75:65-74

27. Desnick RJ, Brady R, Barranger J, Collins AJ, Germain DP, Goldman M, Grabowski G, Packman S, Wilcox WR (2003) Fabry disease, an under-recognized multisystemic disorder: expert recommendations for diagnosis, management, and enzyme replacement therapy. Ann Intern Med 138:338-346

28. Rankin J (1957) Cerebral vascular accidents in patients over the age of 60. II. Prognosis. Scott Med J 2:200-215

29. The EuroQol Group (1990) EuroQol-a new facility for the measurement of health-related quality of life. Health Policy 16:199-208

30. Whybra C, Bähner F, Baron K (2006) Measurement of disease severity and progression in Fabry disease. In: Mehta A, Beck M, Sunder-Plassmann $G$ (eds) Fabry disease: perspectives from 5 years of FOS. Oxford Pharmagenesis, Oxford, pp 315-322

31. Lidove O, Joly D, Barbey F, Bekri S, Alexandra JF, Peigne V, Jaussaud R, Papo T (2007) Clinical results of enzyme replacement therapy in Fabry disease: a comprehensive review of literature. Int J Clin Pract 61:293-302

32. Schaefer RM, Tylki-Szymanska A, Hilz MJ (2009) Enzyme replacement therapy for Fabry disease: a systematic review of available evidence. Drugs 69:2179-2205

33. Eng CM, Germain DP, Banikazemi M, Warnock DG, Wanner C, Hopkin RJ, Bultas J, Lee P, Sims K, Brodie SE, Pastores GM, Strotmann JM, Wilcox WR (2006) Fabry disease: guidelines for the evaluation and management of multi-organ system involvement. Genet Med 8:539-548

34. Lubanda JC, Anijalg E, Bzdúch V, Thurberg BL, Bénichou B, Tylki-Szymanska A (2009) Evaluation of a low dose, after a standard therapeutic dose, of agalsidase beta during enzyme replacement therapy in patients with Fabry disease. Genet Med 11:256-264

35. Banikazemi M, Bultas J, Waldek S, Wilcox WR, Whitley CB, McDonald M, Finkel R, Packman S, Bichet DG, Warnock DG, Desnick RJ, Fabry Disease Clinical Trial Study Group (2007) Agalsidase-beta therapy for advanced Fabry disease: a randomized trial. Ann Intern Med 146:77-86

36. Hughes DA, Elliott PM, Shah J, Zuckerman J, Coghlan G, Brookes J, Mehta AB (2008) Effects of enzyme replacement therapy on the cardiomyopathy of Anderson-Fabry disease: a randomised, double-blind, placebo-controlled clinical trial of agalsidase alfa. Heart 94:153-158

37. Parini R, Rigoldi M, Santus F, Furlan F, De Lorenzo P, Valsecchi G, Concolino D, Strisciuglio P, Feriozzi S, Di Vito R, Ravaglia R, Ricci R, Morrone A (2008) Enzyme replacement therapy with agalsidase alfa in a cohort of Italian patients with AndersonFabry disease: testing the effects with the Mainz Severity Score Index. Clin Genet 74:260-266
38. Spinelli L, Pisani A, Sabbatini M, Petretta M, Andreucci MV, Procaccini D, Lo Surdo N, Federico S, Cianciaruso B (2004) Enzyme replacement therapy with agalsidase beta improves cardiac involvement in Fabry's disease. Clin Genet 66:158-165

39. Hajioff D, Hegemann S, Conti G, Beck M, Sunder-Plassmann G, Widmer U, Mehta A, Keilmann A (2006) Agalsidase alpha and hearing in Fabry disease: data from the Fabry Outcome Survey. Eur J Clin Invest 36:663-667

40. Hilz MJ, Brys M, Marthol H, Stemper B, Dütsch M (2004) Enzyme replacement therapy improves function of C-, Adelta-, and Abeta-nerve fibers in Fabry neuropathy. Neurology 62:10661072

41. Hoffmann B, Beck M, Sunder-Plassmann G, Borsini W, Ricci R, Mehta A, European Investigators FOS (2007) Nature and prevalence of pain in Fabry disease and its response to enzyme replacement therapy-a retrospective analysis from the Fabry Outcome Survey. Clin J Pain 23:535-542

42. Schiffmann R, Floeter MK, Dambrosia JM, Gupta S, Moore DF, Sharabi Y, Khurana RK, Brady RO (2003) Enzyme replacement therapy improves peripheral nerve and sweat function in Fabry disease. Muscle Nerve 28:703-710

43. Schiffmann R, Hauer P, Freeman B, Ries M, Scott LJ, Polydefkis M, Brady RO, McArthur JC, Wagner K (2006) Enzyme replacement therapy and intraepidermal innervation density in Fabry disease. Muscle Nerve 34:53-56

44. Cousins A, Lee P, Rorman D, Raas-Rothschild A, Banikazemi M, Waldek S, Thompson L (2008) Home-based infusion therapy for patients with Fabry disease. Br J Nurs 17:653-657

45. Hughes DA, Mlilligan A, Mehta A (2007) Home therapy for lysosomal storage disorders. Br J Nurs 16:1384, 1386-1389

46. Linthorst GE, Vedder AC, Ormel EE, Aerts JM, Hollak CE (2006) Home treatment for Fabry disease: practice guidelines based on 3 years experience in The Netherlands. Nephrol Dial Transplant 21:355-360

47. Filling-Katz MR, Merrick HF, Fink JK, Miles RB, Sokol J, Barton NW (1989) Carbamazepine in Fabry's disease: effective analgesia with dose-dependent exacerbation of autonomic dysfunction. Neurology 39:598-600

48. Ries M, Mengel E, Kutschke G, Kim KS, Birklein F, Krummenauer F, Beck M (2003) Use of gabapentin to reduce chronic neuropathic pain in Fabry disease. J Inherit Metab Dis 26:413-414

49. Schreiber W, Udvardi A, Kristoferitsch W (2007) Chronic meningitis and lacunar stroke in Fabry disease. J Neurol 254:14471449

50. Caraceni A, Mendoza TR, Mencaglia E, Baratella C, Edwards K, Forjaz MJ, Martini C, Serlin RC, de Conno F, Cleeland CS (1996) A validation study of an Italian version of the Brief Pain Inventory (Breve Questionario per la Valutazione del Dolore). Pain 65:87-92

51. Melzack R (1987) The short-form McGill Pain Questionnaire. Pain 30:191-197 